\title{
EFFECT OF INFARCT SIZE LIMITATION BY PROPRANOLOL ON VENTRICULAR ARRHYTHMIAS AFTER MYOCARDIAL INFARCTION
}

\author{
James R. Stewart,* John K. Gibson, $\dagger$ and \\ Benedict R. Lucchesi $\dagger$ \\ Departments of Internal Medicine ${ }^{*}$ and Pharmacology $\dagger$ \\ University of Michigan Medical Center \\ Ann Arbor, Michigan 48109
}

Pharmacologic interventions designed to preserve ischemic myocardium have been the subject of extensive basic and clinical investigation over the past 10 years. ${ }^{1-3}$ Whereas most of these studies have been concerned with the concept of feasibility of reduction of infarct size by a variety of means, none has looked systematically at the long-term sequelae of such interventions, particularly with regard to late-phase arrhythmias and sudden death. The purpose of this paper is to briefly review the rationale behind infarct size reduction and then to present some experimental data which question the advisability of widespread use of pharmacologic reduction of infarct size as an isolated clinical strategy.

Without attempting an exhaustive review of literature, I have chosen a few clinical studies that I believe are either pro or con the concept of infarct size reduction. Although these studies are not necessarily intended as such, they form the background for our investigations.

The rationale for preserving ischemic myocardium stems from both patho$\operatorname{logic}^{4,5}$ and clinical ${ }^{6}$ studies that show a direct relationship between infarct size and subsequent clinical course with respect to the development of congestive heart failure and cardiogenic shock and also with respect to prognosis. Work by Roberts et al. ${ }^{7}$ and Cox et al. ${ }^{8}$ has shown a direct relationship between infarct size as determined by creatine phosphokinase (CPK) measurements and the frequency of ventricular arrhythmias in the first 20 hours after acute myocardial infarction. These studies suggest that the frequency of early ventricular arrhythmias after acute myocardial infarction reflects the extent of myocardial injury.

Schulze et al. ${ }^{9}$ have investigated the relationship of complex ventricular arrhythmias (Lown Class III-V) 10 to 21 days post infarction to left ventricular dysfunction using Holter monitor recordings and gated blood pool scans. Patients with these late-phase complex ventricular arrhythmias were found to have significantly lower ejection fractions and presumably larger infarcts than did patients without complex ventricular arrhythmias. The overall survival in the group with complex arrhythmias was less than 50\%, despite standard antiarrhythmic therapy.

On the basis of these studies, one could postulate that reduction of infarct size should reduce the incidence of postinfarction arrhythmias, both early and late, and subsequently reduce the tendency to the development of ventricular fibrillation and sudden death.

These studies are just a few of the many investigations that have provided the basis for attempts to limit infarct size. 
Recent studies suggest, however, that in addition to infarct size, areas of jeopardized myocardium play an important role in the etiology of postinfarction arrhythmias. Schaeffer and Cobb ${ }^{10}$ have reported the Seattle experience with survivors of out-of-hospital ventricular fibrillation. They found that recurrent ventricular fibrillation or sudden death developed primarily in those patients whose initial episode was not associated with myocardial infarction. Despite lack of evidence of myocardial necrosis, this group of patients remains at a high risk for the development of malignant ventricular arrhythmias. This suggests that these patients have an area of myocardium at risk for the development of ischemia-related changes in myocardial activation and repolarization with resultant malignant arrhythmias. In contrast, those patients who have evidence of a completed infarction after resuscitation do not have an area of jeopardized myocardium at risk for subsequent ischemia and malignant arrhythmias.

Recent angiographic studies by Taylor et al. ${ }^{11}$ have identified groups of patients at different degrees of risk for sudden death after infarction. Those patients at low risk for the development of sudden death were those with an occluded vessel leading to an akinetic myocardial segment with all other areas of ventricle showing normal wall motion. The patients at highest risk were those with an occluded or partially narrowed vessel supplying the hypokinetic myocardial segment. This suggests that patients with a completed infarct who do not have an area of jeopardized myocardium fare better from the standpoint of sudden death than do those patients with a segment of myocardium at risk for subsequent ischemia.

Finally, population studies ${ }^{12,13}$ have shown that patients surviving an episode of subendocardial infarction have a risk of recurrent infarction and sudden death equal to or greater than that of patients with transmural infarction. This again suggests that despite a smaller area of myocardial necrosis, patients with subendocardial infarction have an area of jeopardized myocardium which may lead to recurrent infarction and sudden death.

Thus, on the basis of these studies, one can postulate that reduction of infarct size by pharmacologic intervention, by salvaging viable yet jeopardized myocardium, may increase the risk for the development of malignant arrhythmias and sudden death.

While many pharmacologic agents have been shown to limit the extent of myocardial necrosis resulting from coronary occlusion, propranolol has been consistently effective in reducing infarct size in a variety of animal models ${ }^{\mathbf{1 4}}$ and in two clinical studies. In one clinical study, Norris et al..$^{15}$ gave intravenous propranolol, $0.1 \mathrm{mg} / \mathrm{kg}$, to patients with acute myocardial infarction within 4 hours of the onset of symptoms and this was followed by oral propranolol for several days. These investigators were able to show a decrease in the number of completed ( $Q$ wave) infarcts and lower peak CPK values in the propranololtreated patients than in control patients. So from this study it appears that the concept of infarct size reduction in the clinical setting is feasible.

With this background in mind, we undertook a series of experiments designed to test the hypothesis that pharmacologic reduction of infarct size may be detrimental to electrophysiologic stability after acue myocardial infarction. In choosing an experimental model, we wanted to be able to achieve several goals. First, we wanted to achieve maximal infarct size reduction. Second, most of the earlier experiments that studied the relationship between ventricular arrhythmias and infarct size reduction used anesthetized animals. 
Since anesthesia may have a profound effect on arrhythmia development, we wanted to be able to monitor for arrhythmias in the conscious, unsedated animal. Third, in addition to looking at spontaneous ventricular arrhythmias, we wanted to have another measure of electrical stability in the conscious animal. In order to do this, we wanted a model in which we could attempt to induce ventricular tachycardia by means of programmed electrical stimulation in the conscious dog after myocardial infarction.

In order to achieve maximal infarct size reduction, we performed a 90 minute proximal occlusion of the left anterior descending (LAD) artery, followed by reperfusion through a critical stenosis in anesthetized male mongrel dogs weighing between 10 and $20 \mathrm{~kg}$. Group I animals received pretreatment with intravenous propranolol, $1 \mathrm{mg} / \mathrm{kg}, 30$ minutes before LAD occlusion. Group II animals received an equal volume of saline solution 30 minutes before occlusion and served as controls, and Group IIr animals were shamoperated controls who underwent thoracotomy and electrode placement only. All animals were monitored continuously intraoperatively for determination of electrocardiographic rhythm and blood pressure. The number and complexity of ventricular arrhythmias during the 90 -minute period of LAD occlusion and for 30 minutes after LAD reperfusion were quantitated. After 30 minutes of reperfusion, bipolar electrodes were attached to the left atrium and right ventricular outflow tract to be used for subsequent recording and programmed stimulation studies. The animals were then allowed to recover and subsequently were monitored daily while standing in a sling, unsedated, for a period of 1 hour until the disappearance of spontaneous ventricular arrhythmias. Significant ventricular arrhythmias were arbitrarily defined as being equal to or greater than 10 premature ventricular depolarizations per minute. This enabled us to assess the time course of spontaneous ventricular ectopy in the conscious animal after myocardial infarction. On the first day in which no significant ventricular ectopy was recorded, the animals underwent programmed electrical stimulation studies using the indwelling electrodes. The stimulation protocol consisted of both atrial and ventricular pacing with the introduction of single and double ventricular extrastimuli as well as short bursts of rapid ventricular pacing to a maximal rate of 250 per minute. The animals were then sacrificed and infarct size determined by triphenyl tetrazolium staining and direct gravimetric analysis and expressed as percent of left ventricular mass.

Animals that were pretreated with propranolol developed an infarct that was on the average $20 \%$ of the left ventricular mass and this was significantly smaller $(p<0.005)$ than the infarct size in the control animals, which averaged $30 \%$ of left ventricular mass. However, despite significant infarct size reduction, the time course of spontaneous ventricular arrhythmias after infarction was prolonged in the propranolol-treated animals compared with the control group. Thus, by 72 hours after occlusion, half of the propranololtreated animals still had significant ventricular ectopy, whereas none of the control animals continued to display significant arrhythmias by this time. By 96 hours after occlusion, both groups of animals were quiescent from the standpoint of spontaneous ventricular ectopy. Despite this difference between the two groups in the time course of spontaneous ventricular arrhythmias, there was no difference in the ability to induce ventricular tachycardia by means of programmed stimulation between the two groups.

Of interest, none of the sham-operated control animals exhibited any spontaneous ventricular ectopy nor did they exhibit induced ventricular tachycardia, and none had any evidence of infarction by our staining techniques. 


\section{SUMMARY}

These experiments show that pretreatment with propranolol was able to achieve significant infarct size reduction in this particular animal model of LAD occlusion followed by reperfusion through a critical stenosis. Despite infarct size reduction, the propranolol pretreatment prolonged the time course of spontaneous ventricular arrhythmias after acute myocardial infarction in the conscious dog. In contrast to this, propranolol pretreatment did not appear to protect against inducible ventricular arrhythmias in the late postinfarction period in the conscious dog.

While it is, of course, impossible to extrapolate the results of animal studies to the clinical situation, future clinical studies designed to limit infarct size by pharmacologic intervention should specifically address the question of electrophysiologic instability after myocardial infarction, both in the early and late phases. The possibility exists that pharmacologic limitation of infarct size may preserve viable but potentially jeopardized areas of myocardium, leaving the risk of subsequent malignant arrhythmias and sudden death.

\section{REFERENCES}

1. Gillespie, T. A. \& B. E. Sobel. 1977. A rationale for therapy of acute myocardial infarction. Limitation of infarct size. Advan. Intern. Med. 22: 319-353.

2. CoRDAY, E., ED. 1976. Symposium on the management of jeopardized ischemic myocardium. Am. J. Cardiol. 37: 461-605.

3. Braunwald, E., Ed. 1976. Protection of the Ischemic Myocardium. American Heart Association Monograph 48.

4. Amsterdam, E. A. 1973. Function of the hypoxic myocardium. Am. J. Cardiol. 32: 461-471.

5. Harnaryan, C., M. A. Bennett, B. L. Pentecost, et al. 1970. Quantitative study of infarcted myocardium in cardiogenic shock. Br. Heart J. 32: 728732.

6. Sobel, B. E., G. F. Breshnahan, W. E. Shell, et al. 1972. Estimation of infarct size in man and its relationship to prognosis. Circulation 46: 640648.

7. Roberts, R., A. Husain, H. D. Ambos, et al. 1975. Relation between infarct size and ventricular arrhythmia. Br. Heart J. 37: 1169-1175.

8. Cox, J. R., R. Roberts, H. D. Ambos, et al. 1976. Relations between enzymatically estimated myocardial infarct size and early ventricular dysrhythmia. Circulation 50: I-150-155.

9. Schulze, R. A., H. W. Strauss \& B. Pitt. 1977. Sudden death in the year following myocardial infarction: Relation to late hospital phase VPC's and left ventricular ejection fraction. Am. J. Med. 62: 192-199.

10. Schaffer, W. A. \& L. A. CoBb. 1975. Recurrent ventricular fibrillation and modes of death in survivors of out-of-hospital ventricular fibrillation. $N$. Engl. J. Med. 293: 259-262.

11. Taylor, G., J. O. Humphries, L. Griffith, et al. 1977. Prognosis after acute infarction: $A$ function of angiographically identified myocardial risk segments (abstract). Circulation 56: 562.

12. Cannom, D. S., W. Levy \& L. S. Cohen. 1976. The short and long term prognosis of patients with transmural and nontransmural myocardial infarction. Am. J. Med. 61: 452-458.

13. Scheinman, M. M. \& J. A. Abbotr. 1973. Clinical significance of transmural 
versus nontransmural electrocardiographic changes in patients with acute myocardial infarction. Am. J. Med. 55: 602-607.

14. MAROKo, P. R., J. K. KJekshus, B. E. Sobel, et al. 1971. Factors influencing infarct size following coronary artery occlusion. Circulation 43: 67-82.

15. Norkis, R. M., N. L. SAMmel, et al. 1978. Protective effect of propranolol in threatened myocardial infarction. Lancet: 907-909.

\section{Discussion}

DR. BIGGER: I would like to discuss your remark concerning persons with nontransmural myocardial infarction who are now recognized as being at risk of early reinfarction or death. You attribute this risk to the possibility of the occurrence of arrhythmias, but it seems to me that the risk is just as likely to occur through ischemia or left ventricular dysfunction.

DR. STEwart: I didn't mean to imply a primary arrhythmic etiology, but I did mean to imply that without a transmural infarction, there is an area of myocardium presumably in jeopardy for ischemia-related reinfarction or ischemia-related arrhythmic changes.

DR. BIGGER: Do all beta adrenergic blocking agents have the same effects as those you showed with propranolol? Or is it possible that they are not all the same?

Dr. STEwart: There is no way I can answer that since we did not investigate other beta blockers in our study.

DR. SCHwartz: As to the higher incidence of arrhythmias at 72 hours: Is there any difference in heart rate between the two groups? A lower heart rate with propanalol might have favored the appearance of arrhythmias. Also I am concerned about your way of inducing infarction. Reperfusion is very nice, but I wonder whether you are not somehow changing the situation, which may be far different from what happens with complete stenosis. Before extrapolations are made from your studies, it would be good to repeat the study with a more traditional method of coronary arterial occlusion.

DR. STEwart: Your points are well taken. In the clinical situation both effects are probably operative. Certainly, infarctions are due to complete occlusion, but recent angiographic studies of infarcts have shown reperfusion in patients within hours after acute infarction.

DR. MASERI: I want to make two points. First, you were very careful to point out that you were describing the results in your model in terms of reducing infarct size. What happens in daily life with patients may be slightly different from what happens to an experimental animal whose artery is occluded and perhaps released.

Second, it is very important, when looking at sudden death after myocardial infarction, to determine whether these sudden deaths are related to arrhythmias per se or to a new episode of ischemia, because if they are related to a new episode of ischemia, then we must strive to prevent such an occurrence. Conversely, if they are related to primarily electrical instability, then antiarrhythmic drugs may have a place. The distinction between causes must be made.

DR. STEWART: I agree with you completely. The main emphasis should be placed on preventing subsequent ischemia. 
Dr. LOzNER: Dr. Pitt's earlier review made the point that survival after myocardial infarction really depends on how much damage has been done to the ventricle. The cumulative effects of multiple infarctions identify the patients who do not do well. Nobody has mentioned yet that we have an exciting clinical tool on the horizon for limiting infarct size by lysing coronary thrombi within hours of the infarction. Although those patients may be at early risk of arrhythmias, especially at the time of reperfusion, a patient whose infarct has been markedly limited by that technique is going to do better over the long term than will someone who just sits and hopes for one of the "good" kinds of infarction, which is a nice neat package with no ischemic area.

Dr. Stewart: I have two comments on that: First, the streptokinase studies may be more relevant to our reperfusion models. Second, patients who receive streptokinase will continue to have a high-grade stenosis in the vessel that was initially responsible for the infarction. If nothing is done about the residual high-grade stenosis, the person is again at a high risk of subsequent infarction. 